\title{
Cautionary note on the use of Caenorhabditis elegans to study muscle phenotypes caused by mutations in the human MYH7 gene
}

\author{
Alejandro Gil-Gálvez ${ }^{1}$, Pilar Carbonell-Corvillo ${ }^{2}$, Carmen Paradas*,2 \& Antonio Miranda-Vizuete*,1 iD \\ ${ }^{1}$ Redox Homeostasis Group, Instituto de Biomedicina de Sevilla (IBIS), Hospital Universitario Virgen del Rocío/CSIC/Universidad de Sevilla, 41013 Sevilla, Spain; \\ ${ }^{2}$ Neuromuscular Unit, Department of Neurology, Instituto de Biomedicina de Sevilla (IBIS), Hospital Universitario Virgen del Rocío/CSIC/Universidad de Sevilla, 41013 \\ Sevilla, Spain; *Author for correspondence: cparadas@us.es; amiranda-ibis@us.es
}

BioTechniques 68: 297-299 (June 2020) 10.2144/btn-2020-0012

First draft submitted: 28 January 2020; Accepted for publication: 18 March 2020; Published online: 16 April 2020

\section{ABSTRACT}

Mutations in the human MYH7 gene, encoding a slow skeletal muscle/ $\beta$-cardiac myosin heavy chain, cause different types of myopathies. The nematode model Caenorhabditis elegans has frequently been employed to study the molecular and physiological consequences of $\mathrm{MYH7}$ mutations in muscle function by introducing mutations into the unc-54 gene, the worm MYH7 ortholog. We report here that the $\mathrm{C}$. elegans model is not appropriate for such studies if they involve expression of the UNC-54 protein (wild-type or fused to green fluorescent protein) above endogenous levels.

KEYWORDS:

Caenorhabditis elegans • mutation $\bullet$ MYH7 • myosin • overexpression • UNC-54

In 1974, Sydney Brenner published his groundbreaking work on the genetic characterization of Caenorhabditis elegans [1]. Since then, this nematode has consolidated as a powerful model organism, not only generating key findings in many different biological disciplines but also serving as a valuable preclinical model for several human diseases, including neuromuscular disorders [2-5]. In his pioneering study, Brenner identified and characterized several classes of mutant based on their visible phenotypes, with the largest gene class being defined by mutants with movement defects, generically designated as 'uncoordinated' (the 'unc' phenotype) [1]. Mutations in one such gene, unc-54, were found to cause body wall muscle cell defects that resulted in worm paralysis $[1,6]$. Subsequent work demonstrated that unc-54 encodes one of the four skeletal muscle myosin heavy chain proteins in this organism [1,6]. unc-54 is one of the most extensively studied genes in $C$. elegans, with hundreds of mutants available and phenotypes spanning from very mild motility defects to lethality, due to the requirement of a functional UNC-54 protein for muscle thick filament assembly [7-9].

unc-54 is the worm ortholog of the human myosin gene $M Y H 7$, mutations in which cause an important group of muscular diseases with variable clinical and morphological expression depending on the mutated isoform and the type and location of the mutation [10]. Slow skeletal muscle/ $\beta$-cardiac myosin heavy chain, encoded by the $M Y H 7$ gene, is expressed in cardiac muscle and all slow skeletal muscle fibers [11]. MYH7 mutations are an established cause of hypertrophic or dilated cardiomyopathy [12], Laing distal myopathy [13] and myosin storage/hyaline body myopathy [14] as well as additional myopathy subtypes.

Laing distal myopathy is an autosomal dominant disease commonly characterized by slowly progressive distal weakness, typically involving ankle dorsiflexor muscles and long toe extensor muscles, causing steppage and the typical 'hanging big toe' sign [13]. However, highly variable clinical and histological phenotypes have been widely reported $[15,16]$, and the same mutation can cause different clinical pictures (age of onset, weakness pattern and severity), even within the same family.

Due to the high amino acid sequence conservation between worm UNC-54 and human MYH7 proteins ( $51 \%$ identity and $71 \%$ similarity) and its transparency throughout the life cycle, the $C$. elegans model has frequently been used to recreate the phenotypic consequences of $\mathrm{MYH7}$ mutations in muscle cell function and morphology. In some cases, transgenic animals expressing an unc-54 gene harboring a human $\mathrm{MYH7}$ mutation are used to evaluate worm motility phenotypes [17]. In other cases, the human $\mathrm{MYH7}$ mutation is incorporated in a transgene expressing an UNC-54::GFP (green fluorescent protein) fusion protein. This tagged transgene can be employed to visualize in vivo the impact of mutations on the integrity of worm muscle cells by labeling the myofilament distribution along the longitudinal cell axis, and to assess the effect of the mutation on worm motility $[18,19]$. We followed this second approach to further characterize a newly identified heterozygous MYH7 founder mutation causing Laing distal myopathy in two apparently unrelated families from southern Spain in which a c.4679G > C transversion generates a p.R1560P amino acid change in the mid-rod region of the MYH7 protein (Figure 1A) [20]. The arginine residue at position 1560 , mutated to proline in the families analyzed in this study, is conserved in worm UNC-54 and is located 
(A)

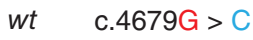

$(\mathrm{CGG} \rightarrow \mathrm{CCG})$
H. sapiens MYH7
1545

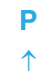
1575
EEAEASLEHEEGK I LR AQLEFNQ I KAE I ERK
$:||||:|||||:|:||||:|\quad:| \mid::$ : || $\mid::$
C. elegans UNC-54
DEAEAALEAEESKVLRAQVEVSQIRSE I EKR 1554

$$
\begin{aligned}
& (\mathrm{CGC} \rightarrow \mathrm{CCC}) \\
& w t \quad \mathrm{c.4706G}>\mathrm{C}
\end{aligned}
$$

(B)
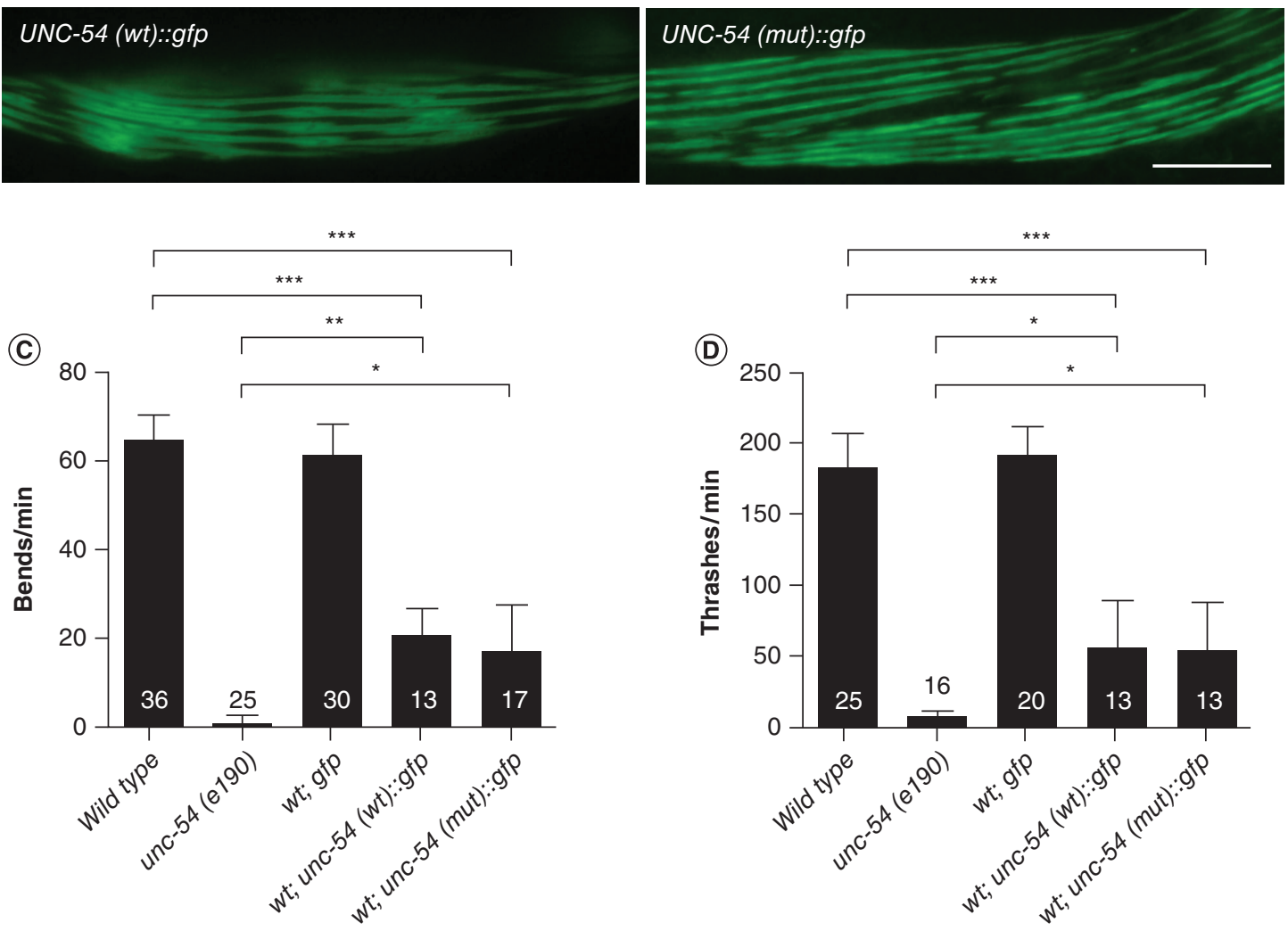

Figure 1. Functional analysis of the human c.4679G $>C$ MYH7 mutation in C. elegans. (A) Schematics of the amino acid residue change between the human MYH7 protein and the orthologous $C$. elegans protein UNC-54. Numbers indicate the amino acid residue of the respective proteins. The wild-type arginine residue is depicted in red and the change to proline is highlighted in blue; the corresponding mutations are shown in parentheses with the same color code. (B) Fluorescence microscopy images showing a normal myofibril distribution in C. elegans transgenic worms expressing UNC-54(wt)::GFP and UNC-54(mut)::GFP fusion proteins. Scale bar $20 \mu \mathrm{m}$. (C, D) Quantification of muscle function performance, measured as the numbers of body bends in solid medium (C) and the numbers of thrashes in liquid medium (D). Data are the mean \pm SD from two independent experiments and the total number of individuals scored is indicated at the base of the column for each genotype ( ${ }^{*} \mathrm{p}<0.05 ;{ }^{* *} \mathrm{p}<0.01$; ${ }^{* \star *} \mathrm{p}<0.001$ by unpaired, two-tail $t$-test). GFP: Green fluorescent protein; mut: Mutation; SD: Standard deviation; wt: Wild type.

at position 1569 (Figure 1A). Previous studies have shown that introducing proline residues in the MYH7 protein interferes with myosin self-assembly [18,21]. Moreover, most MYH7 mutations associated with distal myopathy are located in the mid-rod region of the protein, within exons 32-36 [22], and this is where the c.4679G >C mutation lies [20]. To test whether the p.R1460P change also causes such alteration, we expressed in worm muscle cells an UNC-54::GFP fusion protein with a corresponding proline change to that identified in the patients (UNC-54(mut)::GFP) (Figure 1A). Because the human mutation has an autosomal dominant inheritance pattern, we generated transgenic animals expressing UNC-54(mut)::GFP in a wild-type genetic background; worms expressing GFP alone and UNC-54(wt)::GFP fusion protein in the same wild-type background served as controls. Transgenic UNC-54(mut)::GFP animals were indistinguishable from wild-type, GFP or UNC-54(wt)::GFP control worms in terms of their body morphology (not shown) and they had normal muscle cell 
morphology and longitudinal distribution of the myofilaments (Figure 1B), consistent with the mild alteration seen in patients' muscle biopsies [20].

To determine whether the mutation would cause more subtle motility phenotypes, we used the same transgenic worms to perform motility assays in both liquid medium and on seeded plates. The first is carried out by placing worms in a 5- $\mu$ l drop of M9 buffer and counting the number of lateral swimming movements (thrashes) [23]; the second quantifies the worms' locomotion rate by counting body bends as they move on the bacterial lawn [24]. To our surprise, when performing the motility assay in liquid medium, we found that worms expressing UNC-54(wt):::GFP or UNC-54(mut)::GFP fusion proteins had a very erratic and irregular thrashing pattern compared with the robust, repetitive pattern showed by wild-type or GFP control animals (Supplementary Movies 1-5). The worms in solid medium on seeded plates also demonstrated motility deficits in UNC-54(wt)::GFP or UNC-54(mut)::GFP worms (not shown). In concordance with these observations, quantification of thrashes in liquid medium and body bends in solid medium showed that UNC-54(wt)::GFP or UNC54(mut)::GFP fusion proteins decreased worm motility threefold, compared with wild-type or GFP-expressing control animals (Figure 1C $\& D)$. As the transgenes used in this study are high copy number, this result suggests that increased levels of UNC-54::GFP protein may negatively affect worm muscle cell functionality without impairing myosin filament assembly or overall cell morphology. Prompted by this apparent deleterious effect of the UNC-54::GFP protein per se - which would invalidate previous studies using the same experimental strategy - we revisited similar experiments done by other groups [17-19,25]. In all these studies, production of transgenic UNC-54 or UNC-54:::GFP protein above wild-type levels effectively impairs motility in C. elegans [17-19,25]. Indeed, we found an older work reporting that very high levels of UNC-54 can even disrupt muscle structure [26]. It should be noted that although there has been some controversy about a possible inhibitory effect of GFP on myosin function [27-29], our data clearly show that GFP overexpression does not have any deleterious impact on worm motility (Figure 1C \& D). Thus, worms expressing GFP can be used as controls in this type of experiment.

Together, we conclude that the results of modeling the pathogenic effects of human MYH7 mutations by evaluating the motility phenotypes of such mutations in worms expressing UNC-54 from high-copy transgenes should be viewed with caution. Regardless of whether they are integrated in the genome or maintained extrachromosomally, these transgenes may cause anomalous results by interfering with normal muscle cell function and should therefore be avoided. Effective alternatives to overcome this limitation could come from the use of unc-54 single-copy transgenes or direct CRISPR/Cas 9 editing of the endogenous unc-54 gene. These approaches would more faithfully reproduce the MYH7 mutations of patients in worms with unc-54 wild-type gene dosage.

The $C$. elegans strains used in this study were N2, wild-type Bristol and CB190, unc-54(e190) I. Nematode strains were grown and maintained at $20^{\circ} \mathrm{C}$ on nematode growth medium agar plates seeded with E. coli OP50 as food source [30]. The pPD95.77 plasmid derivative containing the unc-54 promoter plus the genomic ORF fragment that generates an UNC-54::GFP fusion protein [19], a kind gift from Prof. Andras Malnasi-Csizmadia, was used as bait to generate the mutant variant identified in this study with the following mutagenic primers: 5'-GAGCAAGGTTCTCCCCGCCCAGGTTGAAG-3' (forward) and 5'-CTTCAACCTGGGCGGGGAGAACCTTGCTC-3' (reverse). Mutagenesis was performed using the QuickChange II XL Site Directed Mutagenesis Kit (Stratagene) following manufacturer instructions and confirmed by sequencing in both orientations. Wild-type and mutated pPD95.77/unc-54 plasmids were transformed into DH5 $\alpha$ bacteria, purified using the NucleoSpin Plasmid kit (Macherey-Nagel) and injected into the gonad of wild-type worms at a concentration of $20 \mathrm{ng} / \mu \mathrm{l}$. Transgenic progeny were identified by GFP expression in worm muscle cells and stably transmitting lines were used in the experiments. Transgenic animals were mounted in a $5-\mu$ drop of $10 \mathrm{mM}$ levamisole (Sigma) on a microscope slide with a $3 \%$ agarose pad covered with a $24 \times 24 \mathrm{~mm}$ coverslip and the muscle cells' GFP was scored on an Olympus BX61 fluorescence microscope. To assay the motility, well-fed L4 animals were transferred to fresh seeded plates and incubated further for $24 \mathrm{~h}$ at $20^{\circ} \mathrm{C}$ prior to assaying their motility parameters. Then, worms were gently placed on seeded 60-mm plates (solid medium motility) or in a $5 \mu /$ drop of M9 buffer on a glass slide (liquid medium motility); after acclimation for $1 \mathrm{~min}$, the number of bends (solid) or thrashes (liquid) were recorded during the following 2 min using a Leica M165FC stereoscope equipped with a CCD camera (Greenlight Solutions).

\section{Supplementary data}

To view the supplementary data that accompany this paper please visit the journal website at: www.futurescience.com/doi/suppl/10.2144/btn-2020-0012

\section{Author contributions}

A Gil-Gálvez performed all the experiments. P Carbonell-Corvillo and A Miranda-Vizuete designed the experiments. C Paradas and A Miranda-Vizuete wrote the manuscript.

\section{Acknowledgments}

We thank Prof. Andras Malnasi-Csizmadia, Prof. Shu G Chen and the Caenorhabditis Genetic Center for providing the worm strains and plasmids used in this study and Prof. Nigel Laing for helpful discussions and comments on the manuscript.

\section{Financial \& competing interests disclosure}

C Paradas was supported by grants from the Health Institute Carlos III and FEDER (FIS PI16-01843) and the Consejería de Salud, Junta de Andalucía (PI-0085-2016). A Miranda-Vizuete was supported by the Spanish Ministry of Economy and Competitiveness (BFU2015- 
64408-P). The authors have no other relevant affiliations or financial involvement with any organization or entity with a financial interest in or financial conflict with the subject matter or materials discussed in the manuscript apart from those disclosed.

No writing assistance was utilized in the production of this manuscript.

\section{Open access}

This work is licensed under the Attribution-NonCommercial-NoDerivatives 4.0 Unported License. To view a copy of this license, visit http://creativecommons.org/licenses/by-nc-nd/4.0/

\section{References}

1. Brenner S. The genetics of Caenorhabditis elegans. Genetics 77(1), 71-94 (1974).

2. Culetto E, Sattelle DB. A role for Caenorhabditis elegans in understanding the function and interactions of human disease genes. Hum. Mol. Genet. 9(6), 869-877 (2000).

3. Giacomotto J, Segalat L, Carre-Pierrat M, Gieseler K. Caenorhabditis elegans as a chemical screening tool for the study of neuromuscular disorders. Manual and semi-automated methods. Methods 56(1), 103-113 (2012).

4. Brouilly N, Lecroisey C, Martin E et al. Ultra-structural time-course study in the $C$. elegans model for Duchenne muscular dystrophy highlights a crucial role for sarcomere-anchoring structures and sarcolemma integrity in the earliest steps of the muscle degeneration process. Hum. Mol. Genet. 24(22), 6428-6445 (2015).

5. Apfeld J, Alper S. What can we learn about human disease from the nematode C. elegans? Methods Mol. Biol. 1706, 53-75 (2018).

6. MacLeod AR, Waterston RH, Fishpool RM, Brenner S. Identification of the structural gene for a myosin heavy-chain in Caenorhabditis elegans. J. Mol. Biol. 114(1), 133-140 (1977).

7. Moerman DG, Plurad S, Waterston RH, Baillie DL. Mutations in the unc-54 myosin heavy chain gene of Caenorhabditis elegans that alter contractility but not muscle structure. Cell 29(3), 773-781 (1982).

8. Anderson P, Brenner S. A selection for myosin heavy chain mutants in the nematode Caenorhabditis elegans. Proc. Natl Acad. Sci. USA 81(14), 4470-4474 (1984).

9. Bejsovec A, Anderson P. Myosin heavy-chain mutations that disrupt Caenorhabditis elegans thick filament assembly. Genes Dev. 2(10), 1307-1317 (1988).

10. Tajsharghi H, Oldfors A. Myosinopathies: pathology and mechanisms. Acta Neuropathol. 125(1), 3-18 (2013).

11. Udd B. Distal myopathies. Handb. Clin. Neurol. 86, 215-241 (2007).

12. Seidman JG, Seidman C. The genetic basis for cardiomyopathy: from mutation identification to mechanistic paradigms. Cell 104(4), 557-567 (2001).

13. Laing NG, Laing BA, Meredith C et al. Autosomal dominant distal myopathy: linkage to chromosome 14. Am. J. Hum. Genet. 56(2), 422-427 (1995).

14. Tajsharghi H, Thornell LE, Lindberg C, Lindvall B, Henriksson KG, Oldfors A. Myosin storage myopathy associated with a heterozygous missense mutation in MYH7. Ann. Neurol. 54(4), 494-500 (2003)

15. Muelas N, Hackman P, Luque $\mathrm{H}$ et al. MYH7 gene tail mutation causing myopathic profiles beyond Laing distal myopathy. Neurology 75(8), 732-741 (2010).

16. Tasca G, Ricci E, Penttila S et al. New phenotype and pathology features in MYH7-related distal myopathy. Neuromuscul. Disord. 22(7), 640-647 (2012).

17. Dahl-Halvarsson M, Pokrzywa M, Rauthan M, Pilon M, Tajsharghi H. Myosin storage myopathy in C. elegans and human cultured muscle cells. PLoS ONE 12(1), e0170613 (2017).

18. Buvoli M, Buvoli A, Leinwand LA. Effects of pathogenic proline mutations on myosin assembly. J. Mol. Biol. 415(5), 807-818 (2012).

19. Varkuti BH, Yang Z, Kintses B et al. A novel actin binding site of myosin required for effective muscle contraction. Nat. Struc. Mol. Biol. 19(3), 299-306 (2012).

20. Carbonell-Corvillo P, Tristan-Clavijo E, Cabrera-Serrano M et al. A novel MYH7 founder mutation causing Laing distal myopathy in southern Spain. Neuromuscul. Disord. 28(10), 828-836 (2018).

21. Parry DA, Fraser RD, Squire JM. Fifty years of coiled-coils and alpha-helical bundles: a close relationship between sequence and structure. J. Struct. Biol. 163(3), 258-269 (2008).

22. Lamont PJ, Wallefeld W, Hilton-Jones D et al. Novel mutations widen the phenotypic spectrum of slow skeletal/beta-cardiac myosin (MYH7) distal myopathy. Hum. Mutat. 35(7), 868-879 (2014).

23. Buckingham SD, Sattelle DB. Fast, automated measurement of nematode swimming (thrashing) without morphometry. BMC Neurosci. 10, 84 (2009).

24. Hart A. Behavior. WormBook 2006, ed. The C. elegans Research Community, WormBook, doi/10.1895/wormbook.1.87.1, http://www.wormbook.org

25. Tajsharghi H, Pilon M, Oldfors A. A Caenorhabditis elegans model of the myosin heavy chain lla E706K [corrected] mutation. Ann. Neurol. 58(3), 442-448 (2005).

26. Fire A, Waterston RH. Proper expression of myosin genes in transgenic nematodes. EMBO J. 8(11), 3419-3428 (1989).

27. Agbulut $\mathrm{O}$, Coirault C, Niederlander $\mathrm{N}$ et al. GFP expression in muscle cells impairs actin-myosin interactions: implications for cell therapy. Nat. Methods $3(5)$, 331 (2006).

28. Agbulut $\mathrm{O}$, Huet A, Niederlander N, Puceat M, Menasche P, Coirault C. Green fluorescent protein impairs actin-myosin interactions by binding to the actin-binding site of myosin. J. Biol. Chem. 282(14), 10465-10471 (2007).

29. Resnicow DI, Hooft AM, Harrison BC, Baker JE, Leinwand LA. GFP fails to inhibit actin-myosin interactions in vitro. Nat. Methods 5(3), 212-213 author reply 213-214 (2008).

30. Stiernagle T. Maintenance of C. elegans. The C. elegans Research Community, WormBook. 1-11 (2006). http://www.wormbook.org 